\title{
Path-integral simulation of ice Ih: The effect of pressure
}

\author{
Carlos P. Herrero and Rafael Ramírez \\ Instituto de Ciencia de Materiales de Madrid, Consejo Superior de Investigaciones \\ Cientificas (CSIC), Campus de Cantoblanco, 28049 Madrid, Spain
}

(Dated: June 26, 2018)

\begin{abstract}
The effect of pressure on structural and thermodynamic properties of ice Ih has been studied by means of path-integral molecular dynamics simulations at temperatures between 50 and $300 \mathrm{~K}$. Interatomic interactions were modeled by using the effective q-TIP4P/F potential for flexible water. Positive (compression) and negative (tension) pressures have been considered, which allowed us to approach the limits for the mechanical stability of this solid water phase. We have studied the pressure dependence of the crystal volume, bulk modulus, interatomic distances, atomic delocalization, and kinetic energy. The spinodal point at both negative and positive pressures is derived from the vanishing of the bulk modulus. For $P<0$, the spinodal pressure changes from -1.38 to $-0.73 \mathrm{GPa}$ in the range from 50 to $300 \mathrm{~K}$. At positive pressure the spinodal is associated to ice amorphization, and at low temperatures is found between 1.1 and $1.3 \mathrm{GPa}$. Quantum nuclear effects cause a reduction of the metastability region of ice Ih.
\end{abstract}

PACS numbers: 31.70.Ks, 81.40.Vw, 65.40.De, 71.15.Pd

\section{INTRODUCTION}

Despite the large volume of experimental and theoretical work on condensed phases of water, some of their properties still lack a complete understanding. This is mainly due to the peculiar structure of liquid and solid water, where hydrogen bonds between contiguous molecules give rise to properties somewhat different than those of most known liquids and solids (the so-called water "anomalies") $\underline{\underline{1}-\underline{\underline{4}}}$

In the temperature region between $80 \mathrm{~K}$ and $250 \mathrm{~K}$, ice Ih is the stable phase of water up to a pressure around $0.2 \mathrm{GPa}$ (that increases for increasing temperature). This range may seem rather narrow for some purposes, especially if one compares it with other types of materials different from molecular solids. However, this pressure region is large if one takes into account the nature of the intermolecular interaction in water, which gives rise to the presence of hydrogen bonds. The region of mechanical stability of ice $\mathrm{Ih}$, in which this phase is metastable has been studied both experimentally and by computer simulations. At $80 \mathrm{~K}$, simulations $\mathrm{s}^{\frac{5}{6}}$ have predicted it to be metastable down to a negative pressure (tension) of about $-1.4 \mathrm{GPa}$, whereas for positive pressure (compression) its range of metastability is known to extend up to around $1 \mathrm{GPa}$, where ice Ih transforms into an amorphous phase (the so-called high-density amorphous ice) $\underline{\underline{6}}$ This pressure-induced amorphization of ice Ih is an issue that has received in recent years a considerable amount of attention, along with the existence of different amorphous phases which clearly differ in density $\underline{\underline{7}}^{\underline{\underline{9}}}$

Computer simulation of condensed phases of water at an atomic level has a long history, dating back to around $1970{ }^{10.11}$ Since then, important efforts have been focused on the development and refinement of empirical potentials to describe both liquid and solid phases of water, so that nowadays a large variety of this kind of potentials can be found in the literature $\underline{12-17}$. Many of them as- sume a rigid geometry for the water molecule, and some others include molecular flexibility either with harmonic or anharmonic $\mathrm{OH}$ stretches. In recent years, moreover, simulations of water using ab initio density functional theory (DFT) have been carried out $18-20$ Nevertheless, it turns out that the hydrogen bonds in condensed phases of water are difficult to describe with presently available energy functionals, which causes that some properties cannot be accurately reproduced by DFT calculations. ${ }^{21}$ Some promising advances to improve the description of van der Waals interactions in water within the DFT formalism have been recently presented $\underline{22-24}$

A limitation of ab-initio electronic-structure calculations is that they usually treat atomic nuclei as classical particles, not including quantum effects like zero-point motion. These effects can be taken into account by using harmonic or quasiharmonic approximations for the nuclear motion, but the precision of these approaches is not easily estimated when large anharmonicities are present, as can be the case for light atoms like hydrogen. To take into account the quantum character of atomic nuclei, the path-integral molecular dynamics (or Monte Carlo) approach has proved to be very useful, since in this procedure the nuclear degrees of freedom can be quantized in an efficient manner, thus including both quantum and thermal fluctuations in many-body systems at finite temperatures $\stackrel{25,26}{2}$ Thus, a powerful method can consist in combining DFT to determine the electronic structure and path integrals to describe the quantum motion of atomic nuclei $\stackrel{18,20}{ }$ However, this procedure requires computer resources that would restrict enormously the number of state points that can be considered in actual calculations.

The phase diagram of water is now known up to temperatures and pressures on the order of $1000 \mathrm{~K}$ and hundreds of GPa, respectively. Present-day interatomic potentials are able to predict rather accurately the range of stability corresponding to the different known phases $\underline{27}$ 
It is however important to estimate the influence of quantum nuclear effects on the stability range of the different ice phases. In this line, it is known that such quantum effects change the melting temperature $T_{m}$ of ice Ih at ambient pressure by some degrees, as manifested by the isotope effect on $T_{m} \cdot \underline{28}$ This kind of quantum effects has been less studied for liquid and solid water under external pressure. In particular, the spinodal line for ice Ih at negative pressures (which defines the limit of mechanical stability of this water phase) has been calculated by using classical molecular dynamics simulations. $\frac{5}{-}$ Earlier studies of ice Ih using path-integral simulations have been carried out by using mainly effective potentials, and were focused on structural and dynamic properties of the solid phase,$\frac{17,29-32}{2}$

Quantum nuclear effects become more relevant for light atomic masses, and are expected to be especially important in the case of hydrogen. Then, we pose the question of how quantum effects associated to the lightest atom can influence the structural properties of a solid water phase such as ice Ih, and in particular if these effects are relevant or detectable for the solid at different densities, i.e. under different external pressures. This refers to the crystal volume and interatomic distances, but also to the mechanical stability of the solid. In this context, it is usually assumed that increasing quantum fluctuations monotonically enhances the exploration of the energy landscape. However, in certain regimes an increase in quantum fluctuations can lead to dynamical arrest, as shown for glass formation ${ }^{33}$ A similar mechanism could also retard the transition from crystalline to disordered (amorphous) phases.

In the present paper we study ice Ih by path-integral molecular dynamics (PIMD) simulations at different pressures and temperatures, to assess the range of mechanical stability of this water phase, as well as to analyze its structural properties. Interatomic interactions are described by the flexible q-TIP4P/F model, which has been recently developed and was employed to carry out PIMD simulations of liquid water $\underline{\underline{34}}$ and ice $\mathrm{Ih}$ at ambient pressure $\stackrel{28,35}{ }$

The paper is organized as follows. In Sec. II, we describe the computational method and the model employed in our calculations. Our results are presented in Sec. III, dealing with the pressure dependence of crystal volume, bulk modulus, interatomic distances, atomic delocalization, and kinetic energy of ice Ih. Sec. IV includes a summary of the main results.

\section{COMPUTATIONAL METHOD}

We employ here the PIMD method to obtain equilibrium properties of ice $\mathrm{Ih}$ at different temperatures and pressures. This method is based on an isomorphism between the actual quantum system and a classical one, that appears after a discretization of the quantum density matrix along cyclic paths $\frac{36,37}{2}$ This isomorphism is in fact obtained by replacing each quantum particle by a ring polymer consisting of $L$ (Trotter number) classical particles, connected by harmonic springs with temperatureand mass-dependent force constant. Details on this simulation technique can be found elsewhere $\stackrel{25,26}{=}$ We note that the dynamics employed in this method is fictitious and does not correspond to the actual quantum dynamics of the real particles under consideration, but it is used to effectively sample the many-body configuration space, giving precise results for the equilibrium properties of the quantum system. An alternative way to derive equilibrium properties is the use of Monte Carlo sampling, but this procedure has been found to require for the present problem more computer resources than the PIMD method. A particular advantage of the latter is that in this case the codes can be more readily parallelized, an important factor for efficient use of modern computer architectures.

Simulations of ice Ih have been carried out here in the isothermal-isobaric $N P T$ ensemble ( $N$, number of particles; $P$, pressure; $T$, temperature), which allows us to obtain the equilibrium volume of the solid at given pressure and temperature. We have employed effective algorithms for performing PIMD simulations in this statistical ensemble, as those described in the literature $\underline{38-40}$ Sampling of the configuration space has been carried out at temperatures between $50 \mathrm{~K}$ and $300 \mathrm{~K}$, and pressures in the region of mechanical stability of ice Ih (which at $50 \mathrm{~K}$ corresponds to the range from -1.4 to $1.1 \mathrm{GPa}$ ). Note that we have employed both negative (tension) and positive (compression) pressures in the simulations. For comparison with results of PIMD simulations, some simulations of ice Ih were also carried out in the classical limit, which is obtained in our path integral procedure by setting the Trotter number $L=1$.

Our simulations were carried out on ice Ih supercells with periodic boundary conditions. To check the influence of finite-size effects on the results, we considered orthorhombic supercells of two different sizes. The smaller one included 96 water molecules and had parameters $(3 a, 2 \sqrt{3} a, 2 c)$, where $a$ and $c$ are the standard hexagonal lattice parameters of ice Ih, whereas the larger supercell included 288 molecules and had parameters $(4 a, 3 \sqrt{3} a, 3 c)$. Results obtained for both types of supercells coincided within the error bars due to the statistical uncertainty associated to the simulation method. The flexibility of the simulation cell was taken into account in the NPT simulations by treating the modules of the orthorhombic cell vectors as independent dynamic variables. In the considered supercells, and before the PIMD simulations, proton-disordered ice structures were generated by a Monte Carlo procedure, in such a way that each oxygen atom had two chemically bonded and two H-bonded hydrogen atoms, and with a cell dipole moment close to zero $\stackrel{41}{\underline{-}}$

The interatomic interactions have been modeled by the point charge, flexible q-TIP4P/F model, recently developed to study liquid water, $\stackrel{34}{=}$ and that was subsequently 
employed to study several properties of ice, $\stackrel{28,35}{, 3}$ as well

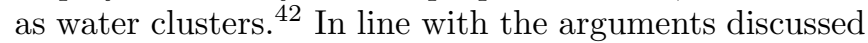
by Habershon et al.,$\underline{34}$ there are some reasons to take this model potential for the present study. First, most previous works that have considered quantum effects in water phases have employed empirical potential models that were parameterized on the basis of earlier classical simulations. Then, quantum simulations using those models lead to "double counting" of quantum effects. Second, many of the empirical potentials previously used for quantum simulations of condensed phases of water treat $\mathrm{H}_{2} \mathrm{O}$ molecules as rigid bodies $\stackrel{30,31,43}{2}$ This can be convenient for computational efficiency, but it disregards the important role of intramolecular flexibility in the structure, dynamics, and thermodynamics of the condensed water phases $\stackrel{34}{\underline{3}}$ Third, the significant anharmonicity of the $\mathrm{O}-\mathrm{H}$ vibration of the water molecule is taken into account by anharmonic stretches in the q-TIP4P/F potential, vs. the harmonic potentials employed in the majority of simulations that considered quantum effects.

Technical details on the simulations presented here are the same as those employed and described in Refs. 28,35. In particular, the Trotter number $L$ was taken proportional to the inverse temperature $(L \propto 1 / T)$, so that $L T$ $=6000 \mathrm{~K}$, which allows one to keep roughly a constant precision in the PIMD results at different temperatures. Also, the time step $\Delta t$ associated to the calculation of interatomic forces was taken in the range between 0.1 and $0.3 \mathrm{fs}$, which was found to provide adequate convergence for the variables studied here. For given temperature and pressure, a typical simulation run consisted of $5 \times 10^{4}$ PIMD steps for system equilibration, followed by $6 \times 10^{5}$ steps for the calculation of ensemble average properties. At ambient pressure, the interatomic potential q-TIP4P/F predicts melting of ice Ih at $251 \mathrm{~K}, 28$ but here we study this water phase up to $300 \mathrm{~K}$, a temperature at which it was found to be metastable along our simulations.

From PIMD simulations one can obtain insight into the atomic delocalization at finite temperatures. This includes a thermal (classical) delocalization, as well as a delocalization associated to the quantum character of the atomic nuclei, which is quantified by the extension of the quantum paths in the path integral formalism. For each quantum path of a given particle, one can define the center-of-gravity (centroid) as

$$
\overline{\mathbf{r}}=\frac{1}{L} \sum_{i=1}^{L} \mathbf{r}_{i}
$$

where $\mathbf{r}_{i}$ is the position of bead $i$ in the associated ring polymer. Then, the mean-square displacement $\Delta_{r}^{2}$ of the atomic nuclei ( $\mathrm{H}$ or $\mathrm{O}$ ) along a PIMD simulation run is defined as

$$
\Delta_{r}^{2}=\frac{1}{L}\left\langle\sum_{i=1}^{L}\left(\mathbf{r}_{i}-\langle\overline{\mathbf{r}}\rangle\right)^{2}\right\rangle
$$

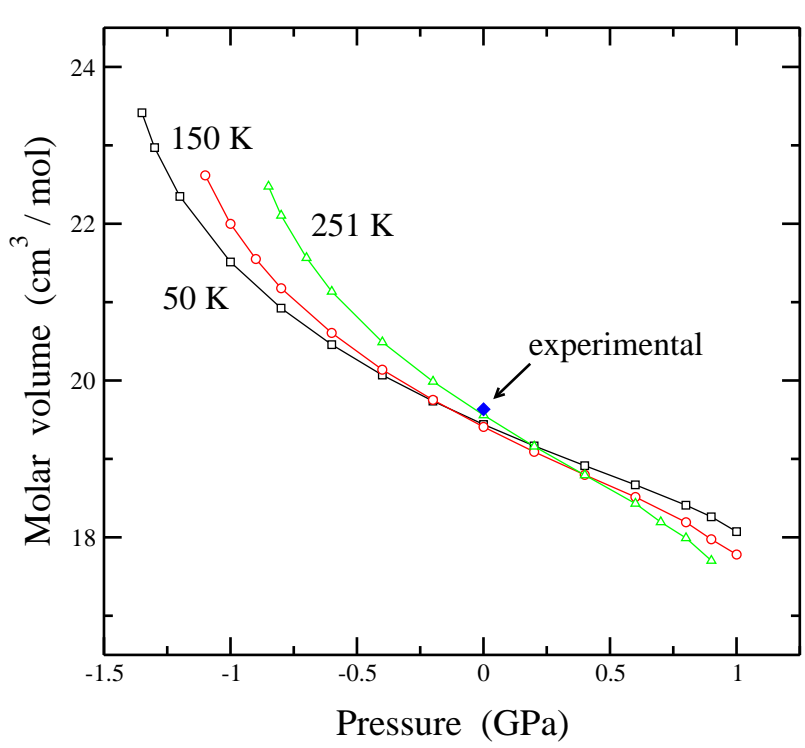

FIG. 1: (Color online) Molar volume of ice Ih as a function of pressure, as derived from PIMD simulations at various temperatures: $50 \mathrm{~K}$ (squares), $150 \mathrm{~K}$ (circles), and $251 \mathrm{~K}$ (triangles). Error bars are in the order of the symbol size. Lines are guides to the eye. A solid diamond represents experimental data from Refs. 4445 at atmospheric pressure and $273 \mathrm{~K}$.

where $\langle\ldots\rangle$ indicates a thermal average at a given temperature. In connection with the quantum delocalization of a particle, in this formalism it is interesting the spread of the paths associated to the particle, which can be measured by the mean-square "radius-of-gyration" $Q_{r}^{2}$ of the ring polymers:

$$
Q_{r}^{2}=\frac{1}{L}\left\langle\sum_{i=1}^{L}\left(\mathbf{r}_{i}-\overline{\mathbf{r}}\right)^{2}\right\rangle .
$$

\section{RESULTS}

\section{A. Volume}

We first present results for the equilibrium volume of ice Ih, as derived from our PIMD simulations at given pressure and temperature, in the pressure region where the solid turned out to be mechanically stable. These results are summarized in Fig. 1, where we show the pressure dependence of the molar volume at three different temperatures: 50, 100, and $251 \mathrm{~K}$. There are several important observations to make with regard to this figure. At a given temperature, we observe the usual volume decrease for rising pressure, i.e., $d V / d P<0$. In most of the pressure region considered, the slope $d V / d P$ becomes less negative as the pressure is raised, which means $d^{2} V / d P^{2}>0$. However, at about $P=0.5 \mathrm{GPa}$ we observe a change in the trend of the first derivative as indicated by the presence of an inflection point with $d^{2} V / d P^{2}=0$. At higher pressures, the slope increases in 
absolute value until reaching the limit of mechanical stability of the solid, close to $P=1 \mathrm{GPa}$. This change in the trend of the $P-V$ curve, with the appearance of an inflection point seems to be related to the proximity of the amorphization of the solid, with an important reduction in the molar volume $e^{5,6,46}$ In fact, this amorphization is associated with a divergence in the compressibility of ice Ih, where the derivative $d V / d P$ should diverge to $-\infty$ (see below).

It is also remarkable that the volume-pressure curves cross at $P \sim 0.2-0.3 \mathrm{GPa}$. At negative pressures we find that the larger volumes correspond to the higher temperatures, as expected for the usual thermal expansion of solids. However, at $P>0$ one finds just the opposite trend: the higher volume corresponds to the lower temperature, a fact associated to the negative thermal expansion occurring for ice Ih at low $T$. The potential TIP4P/F is able to reproduce this negative thermal expansion at atmospheric pressure, as discussed elsewhere. ${ }^{35}$ At $P=$ $1 \mathrm{~atm}\left(10^{-4} \mathrm{GPa}\right)$, the line corresponding to $251 \mathrm{~K}$ in Fig. 1 is still above those found for lower temperatures, but lies below them at higher (but relatively low) positive pressures. This anomalous behavior of the crystal volume as a function of temperature is due to the negative sign of the Grüneisen parameter for TA vibrational modes .46 For a mode in the $n$ 'th phonon branch with wave vector $\mathbf{q}$, this parameter $\gamma_{n}(\mathbf{q})$ is defined from the logarithmic derivative of its frequency with respect to the crystal volume: ${ }^{47}$

$$
\gamma_{n}(\mathbf{q})=-\frac{\partial \ln \omega_{n}(\mathbf{q})}{\partial \ln V}
$$

Negative values of $\gamma_{n}(\mathbf{q})$ for TA modes in ice have been related to the tetrahedral coordination of water molecules, and a negative thermal expansion has been also observed in other solids with similar structures. In particular, it is well known for crystals with diamond and zinc blende structure. $\stackrel{48}{ }$ In connection with this, negative values of $\gamma_{n}(\mathbf{q})$ for TA modes were also found to be related with the pressure-induced amorphization of ice Ih. ${ }^{46}$ In fact, they cause that the relations between elastic constants necessary for crystal stability are violated at a certain applied pressure, giving rise to mechanical instability of the solid. In this line, instabilities in transverse acoustical modes in ice Ih were also found at low temperatures from incoherent inelastic neutron scattering. 49

For $P<0$, the absolute value of the slope $d V / d P$ increases fast, and eventually diverges for a finite value of the pressure, which depends on temperature. This is associated with a divergence of the compressibility of the solid, which gives the limit for the mechanical stability of this ice phase (spinodal point) at negative pressures. On the other side, for positive pressures the stability limit at $P \sim 1 \mathrm{GPa}$ is related to the amorphization of the solid. In fact, at these pressures we find in the PIMD simulations a sudden reduction in the volume of the solid, accompanied by the breaking and reordering of hydrogen bonds (see below). Note that the possibility of studying a solid phase in metastable conditions, close to a spinodal line is limited by the appearance of nucleation events, which cause the breakdown of the crystal structure and the surge of the equilibrium phase. In this kind of atomistic simulations the probability of such nucleation events at low temperatures is relatively low, and the metastable range of the solid that can be explored is rather large. In fact, one can go to conditions close to the spinodal lines at positive and negative pressures, especially at $P<0$.

At $250 \mathrm{~K}$ ice Ih was found to be stable along our simulations up to $P=0.9 \mathrm{GPa}$. At such pressures, the H-bond network of ice Ih collapses and the crystal transforms into the amorphous phase with an important volume decrease. At $T=251 \mathrm{~K}$ and $P=1 \mathrm{GPa}$, this decrease amounts to about $18 \%$ of the crystal volume. Something similar happens at lower temperatures, and at $100 \mathrm{~K}$ we found a volume reduction of $24 \%$ in the amorphization process. This is in line with the pressure-induced amorphization of ice Ih first observed at $T=77 \mathrm{~K}$, and occurring at about $P=1 \mathrm{GPa} . \underline{6}$ This water phase is the so-called high-density amorphous ice ${ }^{7,50}$ Note that at higher $T$ (in the order of $250 \mathrm{~K}$ ) amorphization of ice Ih has not been experimentally observed, and a transition to ice III at $P \sim 0.2 \mathrm{GPa}$ could be expected. Such transitions between crystalline ice phases are usually not observed from the kind of atomistic simulations employed here, and other types of simulations employing thermodynamic integration would be required for this purpose ${ }^{28}$ The important point here is that we obtain at each temperature the pressure range in which ice Ih is metastable with the employed potential model, i.e., we are not discussing the transitions between equilibrium (crystalline) phases, as they would appear in the phase diagram of water.

Our results are in line with those derived from clas-

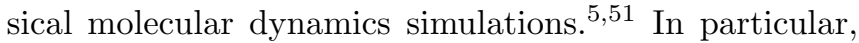
Sciortino et al. $\frac{5}{5}$ employed the TIP $4 \mathrm{P}$ potential, and found that the solid suffers reversible changes at $77 \mathrm{~K}$ for compression $(P>0)$ up to $\sim 1.5 \mathrm{GPa}$. At higher pressures, ice was found to become amorphous in an irreversible way, i.e. it remained in the amorphous phase when decreasing the pressure to low values in the order of $1 \mathrm{~atm}$.

The molar volume of ice Ih has been determined experimentally in different ways, e.g., x-ray, optical, mechanical, calorimetric, acoustical, or nuclear methods (see Ref. 52 and references therein). Measurements of different authors typically deviate from each other by up to about $0.3 \% .52$ Our results at atmospheric pressure are in line with those of Refs. 44, 45, considered in Ref. 52 the most accurate determinations at normal pressure and $T$ $=273 \mathrm{~K}$. These data are represented in Fig. 1 by a solid diamond.

\section{B. Bulk modulus and spinodal pressure}

The compressibility of liquid water and ice shows peculiar properties associated to the hydrogen-bond network 
present in these condensed phases. It turns out that their compressibility is smaller than what one could expect at first sight from the large cavities present in their structure, which could probably collapse under pressure without water molecules approaching close enough to repel each other. However, this is not the case, and for ice Ih in particular the hydrogen bonds holding the crystal structure are known to be rather stable, as revealed by the relatively high pressure necessary to break down the H-bond network. $\underline{\underline{6}}$

The isothermal compressibility $\kappa$ of ice, or its inverse the bulk modulus $\left[B=1 / \kappa=-V(\partial P / \partial V)_{T}\right]$ can be straightforwardly obtained from our PIMD simulations in the isothermal-isobaric ensemble. In this ensemble the isothermal bulk modulus can be calculated from the mean-square fluctuations of the volume, $\sigma_{V}^{2}=\left\langle V^{2}\right\rangle-\langle V\rangle^{2}$, by employing the expression ${ }^{53,54}$

$$
B=\frac{k_{B} T\langle V\rangle}{\sigma_{V}^{2}},
$$

$k_{B}$ being Boltzmann's constant. This expression was used earlier to obtain the bulk modulus of different types of solids from path-integral simulations $.54,55$

At atmospheric pressure we have found results for the bulk modulus of ice Ih similar to those derived from experiments. In particular, at $T=251$ and $300 \mathrm{~K}$, we found $B=9.1 \mathrm{GPa}$ and $8.4 \mathrm{GPa}$, respectively. There appears in the literature a dispersion of data for the isothermal compressibility (or bulk modulus) of ice Ih at the melting temperature and normal pressure. The values of $B$ obtained here are in line with $B=8.49 \mathrm{GPa}$ at $T=273 \mathrm{~K}$, corresponding to the equation of state derived by Feister and Wagner ${ }^{52}$ for this water phase from a set of experimental data. It is difficult to estimate the precision of this value of the bulk modulus due to the lack of error bars for the published data, and the uncertainty may be large, as suggested by the dispersion in experimental data obtained by different authors (see Ref. 52 and references therein). The uncertainty is even larger at lower temperatures, where we could not find any direct experimental data for the isothermal compressibility. The bulk modulus calculated from our PIMD simulations decreases for rising temperature (a fact commonly found in solids), and this behavior appears to be true for all studied pressures, except for $P \gtrsim 1 \mathrm{GPa}$, where this general trend seems to be inverted (see below).

We will first discuss our results for negative pressure (solid under tension). For $P<0$, the bulk modulus decreases as the pressure becomes more negative (see Fig. 2), which means $\partial B / \partial P>0$ at all temperatures under consideration. Then, $B$ is expected to vanish at a temperature-dependent pressure $P_{s}$, which corresponds to the spinodal point defining the limit of mechanical stability of ice Ih at negative pressures (where the compressibility diverges to infinity).

At a given temperature and near the spinodal pressure, our results for the bulk modulus can be fitted to the expression $B \sim\left(P-P_{s}\right)^{1 / 2}$, which allows us to obtain $P_{s}$

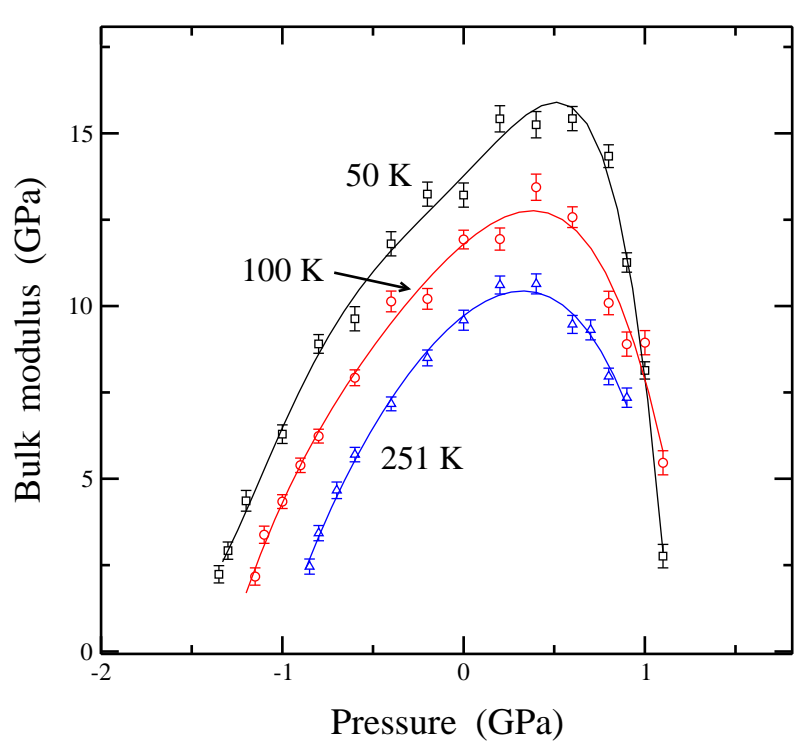

FIG. 2: (Color online) Pressure dependence of the bulk modulus of ice Ih as obtained from quantum PIMD simulations at several temperatures: $50 \mathrm{~K}$ (squares), $150 \mathrm{~K}$ (circles), and 251 $\mathrm{K}$ (triangles). Error bars show the statistical uncertainty in the values of $B$ found from the simulations. Lines are guides to the eye.

from a linear fit of $B^{2}$ vs $P$. This pressure dependence of $B$ can be understood in the following way 56 At a temperature $T$, and calling $F$ the free energy, the spinodal point is given by the condition $\partial^{2} F /\left.\partial V^{2}\right|_{V_{s}}=0$, which can be rewritten as $\partial P /\left.\partial V\right|_{V_{s}}=0$. Thus, close to $P_{s}$ one can write along an isotherm:

$$
P=P_{s}+C_{2}\left(V-V_{s}\right)^{2}+C_{3}\left(V-V_{s}\right)^{3}+\ldots
$$

with constants $C_{2}$ and $C_{3}$ independent of $V$. Assuming $C_{2} \neq 0$ (which seems to be true here), one has $B \approx$ $2 \sqrt{C_{2}} V_{s}\left(P-P_{s}\right)^{1 / 2}$ for small values of the difference $P-$ $P_{s}$ (for discussions on the case $C_{2}=0$, see Ref. 57 59). Such a pressure dependence for $B$, or its equivalent:

$$
P-P_{s} \sim\left(V-V_{s}\right)^{2}
$$

has been obtained earlier for ice and $\mathrm{SiO}_{2}$ cristobalite, as well as for rare-gas solids close to their stability limits,$\frac{5,56}{}$ This means in our case that the exponents describing the singularity are consistent with those of the mean-field spinodal,, 60 given by Eq. (7), where $P_{s}$ and $V_{s}$ are the values of the pressure and volume on the spinodal. Thus, we have obtained $P_{s}$ at each temperature by fitting our results for the bulk modulus at $P<0$ to an expression $B \sim\left(P-P_{s}\right)^{1 / 2}$. The results for $P_{s}$, as derived from our PIMD simulations, are shown in Fig. 3 by solid circles. $P_{s}$ becomes less negative as the temperature is raised, going from $-1.38 \mathrm{GPa}$ at $50 \mathrm{~K}$ to $-0.73 \mathrm{GPa}$ at $300 \mathrm{~K}$.

We now turn to the results at positive pressures (compression). For $P>0$, the bulk modulus increases moderately until reaching a maximum value at about $0.3-0.5$ GPa. This maximum value of $B$ is lower for higher $T$, 


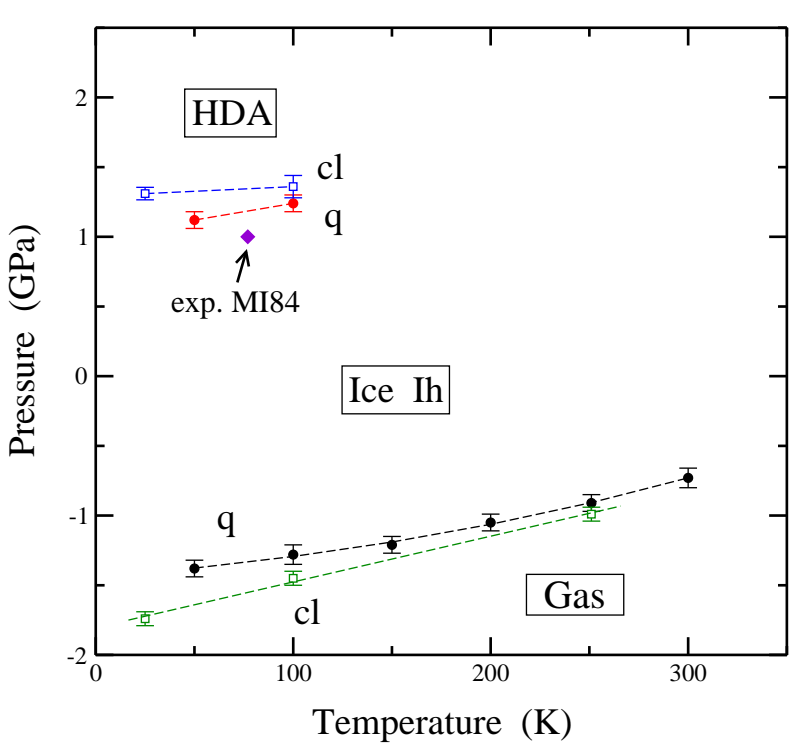

FIG. 3: (Color online) Calculated spinodal pressure for ice Ih as a function of temperature. Solid circles are data obtained from PIMD simulations, whereas open squares represent data yielded by classical simulations. Labels 'q' and 'cl' indicate quantum and classical, respectively. A solid diamond corresponds to the experimental point for amorphization of ice Ih given in Ref. 6 (denoted here as MI84). Labels indicate the regions in the $P-T$ diagram where one finds the different phases: ice Ih, high-density amorphous (HDA), and gas. Lines are guides to the eye.

as shown in Fig. 2, and appears to be slightly shifted to lower pressures as temperature increases. Strässle et al $\underline{61}$ measured the equation of state of ice Ih at $145 \mathrm{~K}$ under hydrostatic pressure up to $0.5 \mathrm{GPa}$, and fitted their results for the pressure dependence of the volume to a Murnaghan equation. They obtained at zero pressure a bulk modulus $B=9.85(47) \mathrm{GPa}$, somewhat lower than that derived from our simulations at $150 \mathrm{~K}: B=11.9 \mathrm{GPa}$.

At pressures larger than $0.5 \mathrm{GPa}$ the bulk modulus derived from our PIMD simulations decreases rather fast, until reaching at each temperature a certain pressure at which $B$ approaches zero and ice Ih becomes mechanically unstable. We will call $P_{s}^{\prime}$ this spinodal pressure. Close to $P_{s}^{\prime}$ one has a relation between $B$ and $P$ similar to that given above, but valid here for $P_{s}^{\prime}>P$, so that: $B \sim\left(P_{s}^{\prime}-P\right)^{1 / 2}$. Fitting our results to this expression we find that the bulk modulus extrapolates to zero for $P_{s}$ $=1.11$ and $1.25 \mathrm{GPa}$, at 50 and $100 \mathrm{~K}$, respectively. At higher temperatures, this extrapolation cannot be done from our PIMD results in a reliable way, since the solid collapses into the amorphous phase at pressures clearly lower than that corresponding to the metastability limit of the crystal, where the bulk modulus is still far from vanishing. This is due to a kinetic phenomenon such as the nucleation of a new phase, which prevents close approach to the spinodal, as indicated above. Thus, at $250 \mathrm{~K}$ ice Ih was metastable in our PIMD simulations only up to $P=0.9 \mathrm{GPa}$, where the bulk modulus is still larger than $7 \mathrm{GPa}$, but the ice crystal transforms into the amorphous phase with an appreciable volume decrease. Arguments equivalent to those presented here for the ice instability associated to a vanishing bulk modulus, have been given in connection with the elastic moduli of this hexagonal crystal. ${ }^{62}$

In Fig. 3 we present values of the spinodal pressure $P_{s}^{\prime}$, as derived from our PIMD simulations, along with those corresponding to negative pressures, $P_{s}$ (represented as solid circles). In both cases the spinodal pressure increases as the temperature is raised. At positive pressure, we show only results up to $100 \mathrm{~K}$, as at higher $T$ the extrapolation employed to determine $P_{s}^{\prime}$ is unreliable, as explained above. These results are compared with those derived from classical molecular dynamics simulations (open squares). At positive pressure, $P_{s}^{\prime}$ derived from classical simulations is larger than that found from PIMD, and the opposite occurs for the spinodal $P_{s}$ at negative pressures. In both cases, quantum and classical results are found to lie closer as temperature is raised. Altogether we find that quantum effects reduce the metastability range of ice Ih. This means that at low temperature the pressure region in which this water phase is metastable is reduced by about $0.3 \mathrm{GPa}$, both at negative and positive pressures. For comparison with the calculated spinodal, we also show in Fig. 3 the value of $P=1 \mathrm{GPa}$ given by Mishima et al $\underline{\underline{6}}$ for the laboratory amorphization pressure at $77 \mathrm{~K}$ (solid diamond). Spinodal pressures for ice Ih have been calculated earlier by Sciortino et al .5 from classical molecular dynamics simulations with the TIP4P potential. These authors found at low temperatures values close to the results of our classical simulations, but at high $T$ they obtained values somewhat higher (less negative) than those found here, e.g., at $250 \mathrm{~K}$ they found $P_{s}=-0.75 \mathrm{GPa}$ vs our value of $-0.99 \mathrm{GPa}$. This difference is presumably due to differences between the interatomic potentials employed in both calculations, as the TIP4P deals with rigid water molecules and the $\mathrm{q}-\mathrm{TIP} 4 \mathrm{P} / \mathrm{F}$ takes into account their flexibility, allowing for bending and stretching of the intramolecular bonds. Simulations of the amorphization of ice Ih using classical molecular dynamics were also carried out by Tse and Klein, $\stackrel{63,64}{\longleftarrow}$ who found ice amorphization at pressures around 1.2-1.3 GPa at temperatures between 80 and $100 \mathrm{~K}$, close to the spinodal $P_{s}^{\prime}$ obtained here.

We remark that values of the bulk modulus calculated from PIMD simulations in the isothermal-isobaric ensemble show relative error bars larger than those obtained for other variables (e.g., molar volume, kinetic energy, or interatomic distances), as a consequence of the statistical uncertainty in the volume fluctuations $\sigma_{V}$, employed to calculate the bulk modulus. An alternative way to derive $B$ consists in calculating numerically the derivative $\partial V / \partial P$ from the $P-V$ equation of state at temperature $T$. We have checked that this method gives results that agree within error bars with those derived from the volume fluctuations through Eq. (5). However, the calcula- 


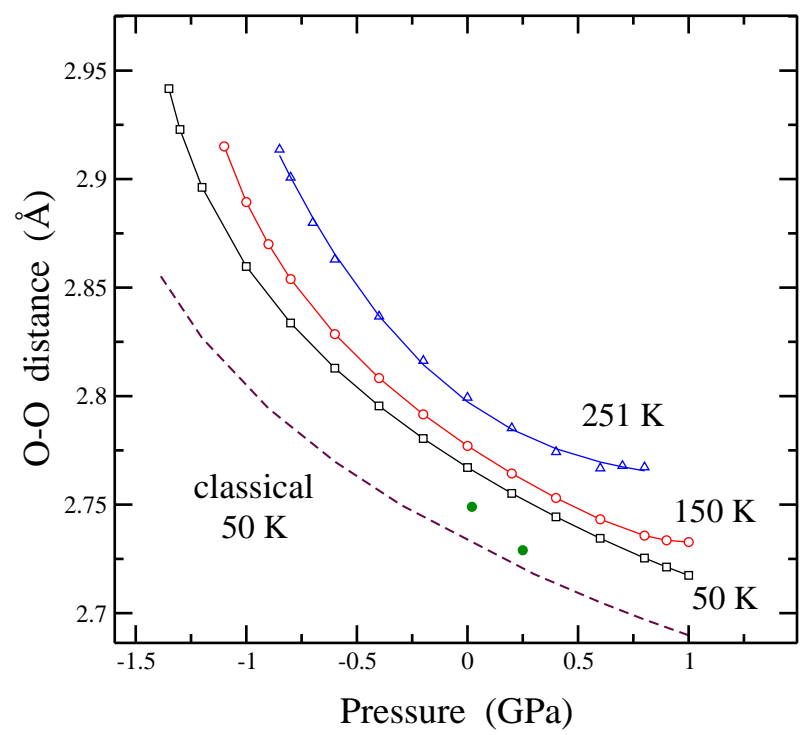

FIG. 4: (Color online) Mean distance between oxygen atoms in nearest-neighbor water molecules in ice Ih, as a function of pressure. Symbols represent results of PIMD simulations at different temperatures: $50 \mathrm{~K}$ (squares), $150 \mathrm{~K}$ (circles), and $251 \mathrm{~K}$ (triangles). Lines are guides to the eye. The dashed line represents the results of classical simulations at $50 \mathrm{~K}$. Solid circles represent $\mathrm{O}-\mathrm{O}$ distances derived from the structural data obtained by Strässle et al. ${ }^{61}$ from neutron diffraction experiments of $\mathrm{D}_{2} \mathrm{O}$ ice $\mathrm{Ih}$ at $145 \mathrm{~K}$.

tion based on the pressure derivative of the volume may be less reliable in the pressure region where $B$ changes fast, as happens close to $P=1 \mathrm{GPa}$ (see Fig. 2).

\section{Interatomic distances}

In this section we show results for interatomic distances in ice Ih between atoms in the same and adjacent molecules. This can shed light on the structural changes suffered by the crystal when temperature and/or pressure are modified. We first show in Fig. 4 the mean distance between oxygen atoms in neighboring molecules as a function of external pressure. Open symbols represent results of PIMD simulations at different temperatures. In each case, we give values of $d(\mathrm{O}-\mathrm{O})$ in the region where the crystal was found to be metastable in the PIMD simulations. This includes, as in previous figures, positive and negative values of $P$. For increasing pressure, $d(\mathrm{O}-$ $\mathrm{O})$ is reduced, as could be expected from the associated volume decrease $(d V / d P<0)$. Nevertheless, contrary to the case of the crystal volume, the isothermal lines corresponding to the $\mathrm{O}-\mathrm{O}$ distance do not cross each other, so that at higher temperature one finds a larger distance in the whole metastability region of ice Ih.

For an homogeneous and isotropic expansion (or contraction) of the crystal, one expects for small volume changes: $\Delta V / V=3 \Delta l / l, l$ being any distance in the solid, and one would expect this relation to be fulfilled, in particular, for the intermolecular distances in the solid. However, although this relation is roughly followed in the parameter range considered here, it is obvious that it cannot be strictly accomplished, given the apparent different trends of the curves shown for crystal volume and $\mathrm{O}-\mathrm{O}$ distance in Figs. 1 and 4 . In particular, volume curves at different temperatures cross each other, whereas those corresponding to the $\mathrm{O}-\mathrm{O}$ distance follow a regular pattern without crossings. The molar volume at low temperature $(T=50 \mathrm{~K})$ decreases by $7.0 \%$ when going from atmospheric pressure to $P=1 \mathrm{GPa}$, from where one would expect a relative change in the average $\mathrm{O}-\mathrm{O}$ distance of $2.3 \%$. This value is somewhat higher than the change actually obtained from the simulations, i.e., $1.8 \%$. On the other side, at negative pressure we find a relative volume increase of $20.4 \%$ at $P=-1.35$ $\mathrm{GPa}$ (close to the spinodal pressure $P_{s}$ ), which would correspond to a relative change in the $\mathrm{O}-\mathrm{O}$ distance of $6.8 \%$. In fact, we find from the simulations an increase in the $\mathrm{O}-\mathrm{O}$ distance of $6.3 \%$, slightly smaller than that expected for an homogeneous and isotropic crystal expansion. This indicates that expanding or contracting the ice crystal is accompanied by a distortion of the tetrahedral network of water molecules. This kind of distortion is also related to the negative thermal expansion of ice Ih occurring at low temperatures, and that was earlier found to be reproduced by PIMD simulations with the q-TIP4P/F potential. 35

For comparison with the results of PIMD simulations, we also show in Fig. 4 results of classical molecular dynamics simulations with the same interatomic potential, which are shown for $T=50 \mathrm{~K}$ by a dashed line. At atmospheric pressure the average $\mathrm{O}-\mathrm{O}$ distance obtained in the quantum simulations is $0.032 \AA$ larger than that derived from classical simulations, which means a difference of $1.2 \%$. This difference increases at negative pressures, and at $P=-1.2 \mathrm{GPa}$ and $T=50 \mathrm{~K}$ we find an increase of $0.069 \AA$ due to quantum effects, i.e., a $2.4 \%$ of the $\mathrm{O}-\mathrm{O}$ distance. These numbers are consistent with the volume increase associated to quantum effects. In fact, at $P=1 \mathrm{~atm}$ the quantum result for the molar volume is $3.4 \%$ larger than the classical one, vs an increase of $7.2 \%$ obtained at $P=-1.2 \mathrm{GPa}$ (see Ref. 35 for results at ambient pressure and several temperatures). In Fig. 4 we also display $\mathrm{O}-\mathrm{O}$ distances derived from the structural data obtained by Strässle et al ${ }^{61}$ from neutron diffraction experiments of $\mathrm{D}_{2} \mathrm{O}$ ice $\mathrm{Ih}$ at $145 \mathrm{~K}$ (solid circles). Note that $\mathrm{O}-\mathrm{O}$ distances in ice may change with the hydrogen isotope, ${ }^{35}$ so that these data for $\mathrm{D}_{2} \mathrm{O}$ ice at 0.02 and $0.25 \mathrm{GPa}$ could differ from those corresponding to $\mathrm{H}_{2} \mathrm{O}$ ice, but in any case they are useful for comparison with our PIMD results.

We now consider the interatomic distances inside water molecules in the ice crystal, as derived from our simulations at different pressures. In Fig. 5 we have plotted the mean intramolecular $\mathrm{O}-\mathrm{H}$ distance as a function of pressure at three temperatures. Concerning this figure, there appear two results that should be emphasized. 


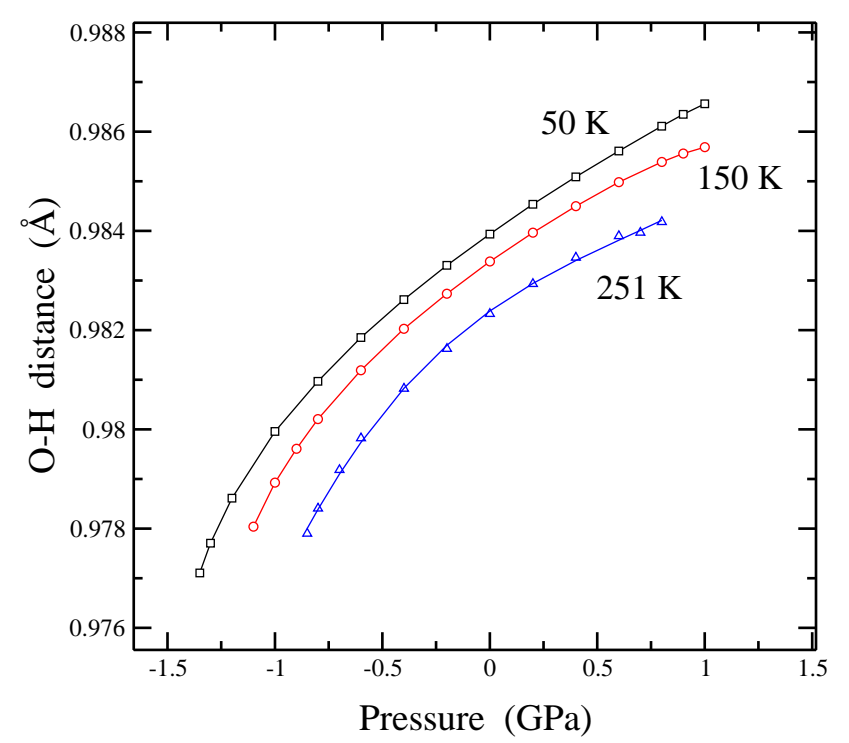

FIG. 5: (Color online) Pressure dependence of the mean intramolecular $\mathrm{O}-\mathrm{H}$ distance, as derived from simulations of ice Ih. Open symbols represent results of PIMD simulations at different temperatures: $50 \mathrm{~K}$ (squares), $150 \mathrm{~K}$ (circles), and $251 \mathrm{~K}$ (triangles). Error bars are in the order of the symbol size. Lines are guides to the eye.

First, at a given pressure we observe that the $\mathrm{O}-\mathrm{H}$ distance decreases as temperature is raised, contrary to the usual expansion of atomic bonds for increasing $T$. Second, at a given temperature, we find that the $\mathrm{O}-\mathrm{H}$ bond distance increases for rising pressure, which may seem also surprising given the pressure-induced volume contraction. Both facts are due to the characteristic structure of ice with hydrogen bonds connecting contiguous water molecules, and can be explained by the same argument: An increase in the intramolecular $\mathrm{O}-\mathrm{H}$ distance is associated to a weakening of the $\mathrm{O}-\mathrm{H}$ bond, and is caused by a hardening of the intermolecular $\mathrm{H}$ bond. For rising $T$, molecular motion is enhanced, so that hydrogen bonds become softer, and the average intermolecular $\mathrm{O}-\mathrm{H}$ distance increases (in line with the rise in $\mathrm{O}-\mathrm{O}$ distance shown above and in Ref. 35). This gives rise to a strengthening of the intramolecular $\mathrm{O}-\mathrm{H}$ bonds, with the associated decrease in interatomic distance in water molecules. This hardening of intramolecular $\mathrm{O}-\mathrm{H}$ bonds for rising temperature has been observed experimentally and reported in the literature $\stackrel{65}{\underline{6}}$ For increasing pressure at a given temperature, we find something similar: the intermolecular $\mathrm{O}-\mathrm{O}$ distance is reduced as the pressure rises (see Fig. 4), in such a way that the intermolecular $\mathrm{H}$ bonds become stronger, causing weaker and longer intramolecular $\mathrm{O}-\mathrm{H}$ bonds. The rise in $\mathrm{O}-\mathrm{H}$ distance derived from our simulations in the range between 0 and $1 \mathrm{GPa}$ is consistent with an increase of about $2 \times 10^{-3}$ $\AA / \mathrm{GPa}$, given in Refs. 66 67 for high-pressure ice phases, although smaller values have been found in Ref. 68 .

Note, however, that the change of intermolecular $\mathrm{O}-\mathrm{O}$

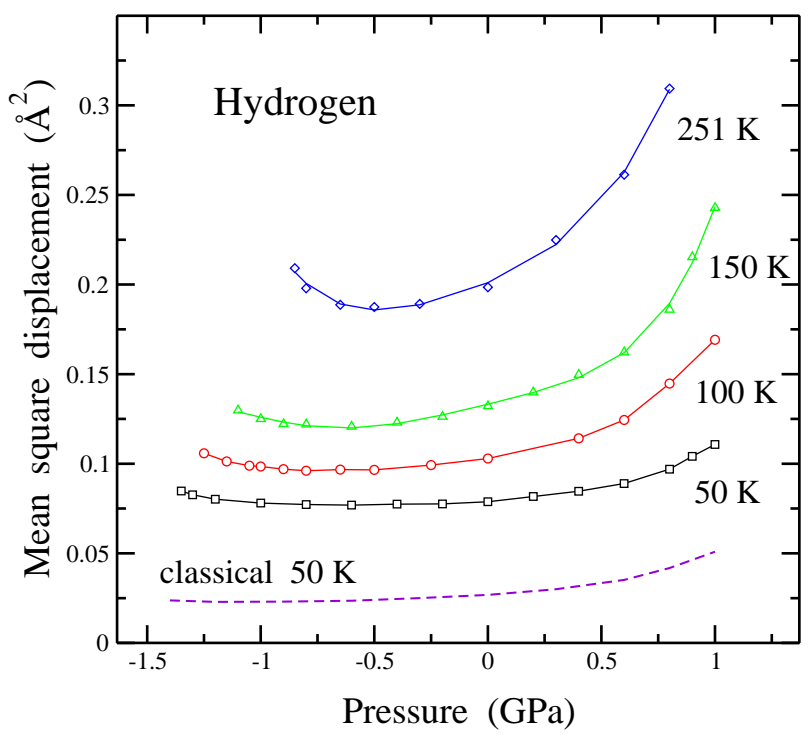

FIG. 6: (Color online) Pressure dependence of the spatial delocalization of hydrogen nuclei (protons) in ice Ih, as derived from PIMD simulations. Symbols indicate the mean-square displacement $\Delta_{r}^{2}$ at several temperatures: $50 \mathrm{~K}$ (squares), 100 $\mathrm{K}$ (circles), $150 \mathrm{~K}$ (triangles), and $251 \mathrm{~K}$ (diamonds). Lines are guides to the eye. The dashed line is the mean-square displacement of hydrogen derived from classical molecular dynamics simulations at $50 \mathrm{~K}$.

distance with pressure is much larger than that of the intramolecular $\mathrm{O}-\mathrm{H}$ distance. Thus, at $50 \mathrm{~K}$ the change in $d(\mathrm{O}-\mathrm{O})$ in the whole metastability region of ice $\mathrm{Ih}$ (from $P=-1.38$ to $1.12 \mathrm{GPa}$ ) is about $8 \%$, whereas the change in intramolecular $\mathrm{O}-\mathrm{H}$ distance is about $1 \%$. This is indeed a consequence of the direct effect of pressure in changing the intermolecular distance, to be compared with an indirect effect on the intramolecular $\mathrm{O}-\mathrm{H}$ bond strength caused by intermolecular $\mathrm{H}$ bonds.

We finally note that the intramolecular $\mathrm{O}-\mathrm{H}$ distance increases appreciably due to quantum nuclear effects. In fact, a classical simulation at $T=50 \mathrm{~K}$ and ambient pressure yields a mean $\mathrm{O}-\mathrm{H}$ distance of $0.969 \AA$, to be compared with $0.984 \AA$ derived from PIMD simulations, which means an increase of $1.5 \%$ in the bond length. This difference is much larger than the temperature-induced change in $d(\mathrm{O}-\mathrm{H})$ shown in Fig. 5. However, the increase due to quantum motion is rather constant in the whole pressure range studied here, i.e. at negative and positive pressures we find a rise in $\mathrm{O}-\mathrm{H}$ distance of about $1.5 \%$.

\section{Atomic delocalization}

Here we present results for the atomic mean-square displacements $\Delta_{r}^{2}$ defined in Eq. (21). One expects that this spatial delocalization will be larger for hydrogen than for oxygen, due to the smaller mass of the former. In Fig. 6 we display values of $\Delta_{r}^{2}$ for hydrogen in ice Ih as a function of pressure. Data were derived from PIMD sim- 


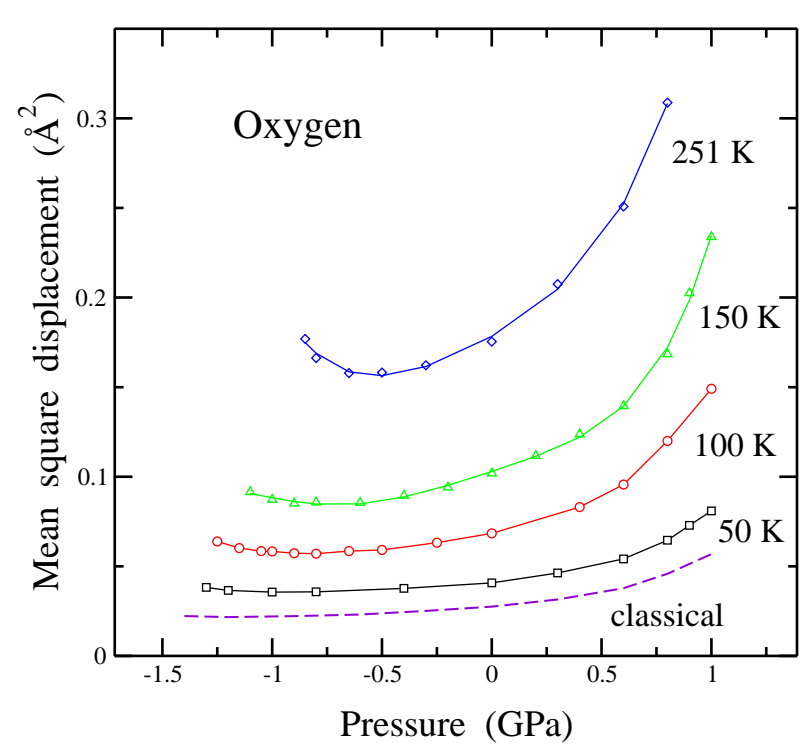

FIG. 7: (Color online) Pressure dependence of the spatial delocalization of oxygen nuclei, as derived from PIMD simulations. Symbols represent the mean-square displacement $\Delta_{r}^{2}$ at several temperatures: $50 \mathrm{~K}$ (squares), $100 \mathrm{~K}$ (circles), 150 $\mathrm{K}$ (triangles), and $251 \mathrm{~K}$ (diamonds). Lines are guides to the eye. The dashed line is the mean-square displacement of oxygen derived from classical molecular dynamics simulations at $50 \mathrm{~K}$.

ulations at different temperatures. At a given pressure, $\Delta_{r}^{2}$ increases as the temperature is raised, in the whole region where the solid is found to be metastable. At 50 $\mathrm{K}$ (the lowest temperature shown here), $\Delta_{r}^{2}$ changes very little as a function of pressure, except close to $P=1 \mathrm{GPa}$, where it increases by a small amount as one approaches the spinodal point (which at this temperature was found to be $P_{s}^{\prime}=1.12 \mathrm{GPa}$; see above). At higher temperatures, changes in $\Delta_{r}^{2}$ as a function of pressure are more appreciable. At all temperatures considered, we find an increase in the atomic delocalization when approaching one of the spinodal points, at positive or negative pressure. The increase at positive pressure (close to amorphization of the solid) is clearly larger than that found at negative pressures. In Fig. 6 we also present the meansquare displacement of hydrogen atoms in Ih, as derived from classical simulations at $50 \mathrm{~K}$ (dashed line). These results are clearly lower than those of the PIMD simulations at the same temperature. Thus, at ambient pressure the classical result is $\Delta_{r}^{2}=2.68 \times 10^{-2} \AA^{2}$, to be compared with $\Delta_{r}^{2}=7.88 \times 10^{-2} \AA^{2}$ found in the quantum simulations.

For oxygen we find mean-square displacements that change with pressure in a way similar to those of hydrogen. This is shown in Fig. 7 at the same temperatures as in Fig. 6 for hydrogen. At atmospheric pressure, we find for oxygen $\Delta_{r}^{2}=4.08 \times 10^{-2} \AA^{2}$ at $50 \mathrm{~K}$ vs 0.175 $\AA^{2}$ at $251 \mathrm{~K}$. Values of $\Delta_{r}^{2}$ are larger for hydrogen than for oxygen, but the difference between both decreases for increasing temperature. Thus, at $T=50 \mathrm{~K}$ and $P=$
$1 \mathrm{~atm}, \Delta_{r}^{2}$ for hydrogen is 1.93 times the mean-square displacement for oxygen, whereas this ratio decreases to 1.13 at $251 \mathrm{~K}$. This is in line with a larger quantum delocalization for hydrogen, caused by its lower mass, and the effect of the mass becomes less relevant as temperature increases.

Note that the mean-square displacement $\Delta_{r}^{2}$ of a given atomic nucleus can be decomposed into a contribution $Q_{r}^{2}$ coming from the spread of the quantum paths [see Eq. (3)], and another one, $C_{r}^{2}$, given by the spatial displacement of the centroid $\overline{\mathbf{r}}$ of the paths $\frac{25,35}{\text { At } 50 \mathrm{~K}}$ and $1 \mathrm{~atm}, Q_{r}^{2}$ represents a $62 \%$ of $\Delta_{r}^{2}$ for hydrogen and a $25 \%$ in the case of oxygen, which reflects the fact that the quantum contribution to the atomic delocalization is more important in the case of hydrogen. This can also be seen by directly comparing values of $Q_{r}^{2}$ for the different atomic species, which result to be 4.8 times larger for $\mathrm{H}$ than for $\mathrm{O}$ at $50 \mathrm{~K}$ and atmospheric pressure.

At low temperatures, the quantum contribution (spreading of the paths), $Q_{r}^{2}$, dominates the spatial delocalization of hydrogen, since the centroid displacement, $C_{r}^{2}$, converges to zero as $T \rightarrow 0 \mathrm{~K}$. The opposite happens in the high-temperature limit (unreachable here for stability reasons), where the quantum contribution $Q_{r}^{2}$ eventually disappears, as corresponds to the classical limit. We note in passing that at low temperatures the quantum paths associated to hydrogen have an average extension of about $0.15 \AA$, much smaller than the $\mathrm{H}-\mathrm{H}$ distance in a water molecule, thus justifying the neglect of quantum exchange between protons in the PIMD simulations.

In Fig. 7 we also show mean-square displacements of oxygen atoms, as derived from classical simulations at $50 \mathrm{~K}$ (dashed line). As one could expect, these values are smaller than those derived from PIMD simulations, but in the case of oxygen the difference between both sets of results, although nonnegligible, is not so important as for hydrogen. In fact, at ambient pressure we found for oxygen $\Delta_{r}^{2}=2.75 \times 10^{-2} \AA^{2}$ in classical simulations vs $4.07 \times 10^{-2} \AA^{2}$ derived from PIMD simulations. Note that the classical result is similar to that found for hydrogen at the same pressure and temperature, but the quantum $\Delta_{r}^{2}$ for $\mathrm{H}$ is clearly larger than that of $\mathrm{O}$ atoms. Also, quantum effects on the meansquare displacement for oxygen decrease rapidly as temperature is raised. Thus, at $T=150 \mathrm{~K}$ and $P=1 \mathrm{~atm}$ we find in the classical simulations $\Delta_{r}^{2}=0.091 \AA^{2}$, to be compared with $\Delta_{r}^{2}=0.102 \AA^{2}$ derived from PIMD simulations, i.e., quantum effects increase $\Delta_{r}^{2}$ by a $12 \%$ at this temperature.

\section{E. Kinetic energy}

The kinetic energy of atomic nuclei in a solid or molecule depends on the mass and delocalization of the considered nucleus. Thus, although in a classical approach, each degree of freedom contributes to the kinetic energy by an amount that depends only on temperature 


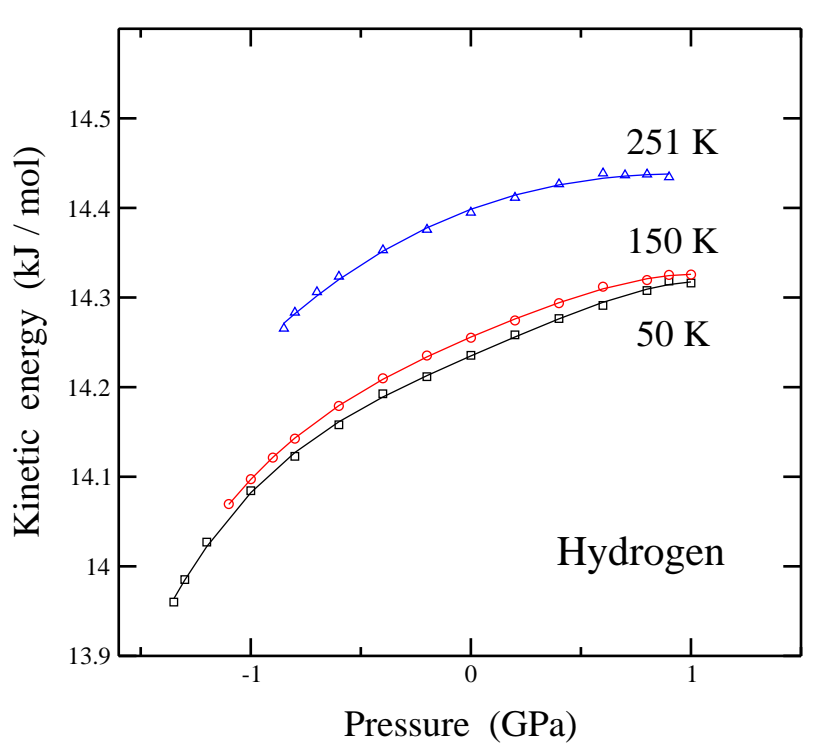

FIG. 8: (Color online) Kinetic energy of hydrogen in ice Ih as a function of pressure. Open symbols indicate results derived from PIMD simulations at different temperatures: $50 \mathrm{~K}$ (squares), $150 \mathrm{~K}$ (circles), and $251 \mathrm{~K}$ (triangles). Error bars are less than the symbol size. Lines are guides to the eye.

( $k_{B} T / 2$, as given by the equipartition principle), in a quantum approach the kinetic energy, $E_{k}$, gives information on the environment and interatomic interactions seen by the considered particle. In particular, a typical quantum effect related to the atomic motion in solids is that the kinetic energy at low temperature converges to a finite value associated to zero-point motion, contrary to the classical result where $E_{k}$ vanishes at $0 \mathrm{~K}$. Path integral simulations allow us to calculate the kinetic energy of the quantum particles under consideration, which is related to the spread of the quantum paths. In fact, for a particle with a certain mass and at a given temperature, the larger the mean-square radius-of-gyration of the paths, $Q_{r}^{2}$, the smaller the kinetic energy, in line with the expectancy that a larger quantum delocalization causes a reduction in the kinetic energy. 25.35 Here we have calculated $E_{k}$ by using the so-called virial estimator, which has an associated statistical uncertainty appreciably lower than the potential energy of the system. ${ }^{39,69}$

We present separately the kinetic energy of oxygen and hydrogen atoms in ice Ih. In Fig. 8 we display $E_{k}$ for hydrogen as a function of pressure at three different temperatures, as derived from our PIMD simulations. At each considered temperature, $E_{k}$ increases as pressure rises, corresponding to an overall increase of vibrational frequencies. $E_{k}$ also increases with temperature, but the data for $T=50$ and $100 \mathrm{~K}$ (not shown in the figure) are almost indistinguishable, since at these temperatures the hydrogen vibrations are nearly in their ground state. Higher vibrational modes are excited at higher temperatures, and at $251 \mathrm{~K}$ we observe an increase in $E_{k}$ larger than $0.1 \mathrm{~kJ} / \mathrm{mol}$ in the whole metastability range of ice

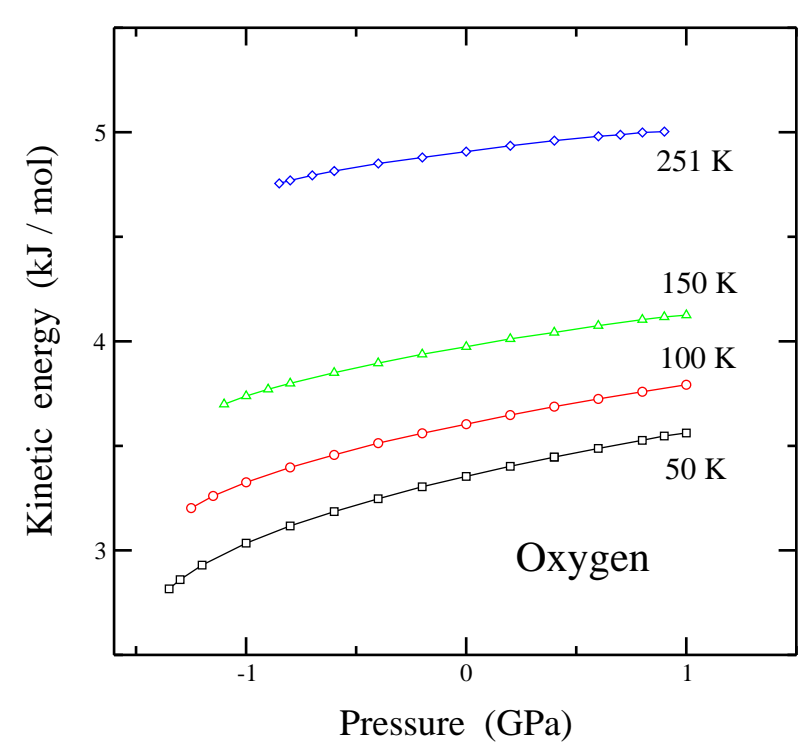

FIG. 9: (Color online) Kinetic energy of oxygen atoms in ice Ih as a function of pressure. Open symbols indicate results derived from PIMD simulations at different temperatures: 50 $\mathrm{K}$ (squares), $100 \mathrm{~K}$ (circles), $150 \mathrm{~K}$ (triangles), and $251 \mathrm{~K}$ (diamonds). Error bars are less than the symbol size. Lines are guides to the eye.

Ih. Values of $E_{k}$ calculated at $P=1$ atm coincide with those presented earlier from PIMD simulations in the range from 210 to $290 \mathrm{~K} \underline{\underline{70}}$

The kinetic energy of hydrogen atoms in ice Ih was obtained by Reiter et al $\stackrel{\text { 71 }}{\underline{1}}$ at ambient pressure from inelastic neutron scattering experiments. These authors found at $269 \mathrm{~K}$ a kinetic energy $E_{k}=12.7 \mathrm{~kJ} / \mathrm{mol}$, somewhat lower than those found here at $P=1 \mathrm{~atm}$ in the temperature range from 250 to $290 \mathrm{~K}$, i.e., $E_{k}$ for hydrogen between 14.39 and $14.44 \mathrm{~kJ} / \mathrm{mol}$.

In Fig. 9 we show the pressure dependence of the kinetic energy of oxygen in ice Ih at four temperatures. As for hydrogen, for oxygen $E_{k}$ also grows for increasing pressure at a given temperature. However, now the isothermal lines at 50 and $100 \mathrm{~K}$ are clearly distinct by more than $0.2 \mathrm{~kJ} / \mathrm{mol}$, as a consequence of the higher mass of oxygen, which produces a lower vibrational energy and an appreciable excitation of vibrational modes at $100 \mathrm{~K}$. From the results for the kinetic energy of $\mathrm{H}$ and O shown in Figs. 8 and 9, we observe that at atmospheric pressure and low temperatures, $E_{k}$ for a hydrogen atom in ice Ih is about 4 times larger than that of an oxygen atom. At $50 \mathrm{~K}$ the kinetic energy of hydrogen increases from $13.96 \mathrm{~kJ} / \mathrm{mol}$ at $P=-1.35 \mathrm{GPa}$ to $14.32 \mathrm{~kJ} / \mathrm{mol}$ for $1 \mathrm{GPa}$. This represents an increase of $0.36 \mathrm{~kJ} / \mathrm{mol}$, i.e., $2.6 \%$ in the whole pressure range. For oxygen, the increase in $E_{k}$ in the same region is about twice that of hydrogen $\left(\Delta E_{k}=0.75 \mathrm{~kJ} / \mathrm{mol}\right)$, but its relative change is much more important for $\mathrm{O}$ (a $26.5 \%$ ).

Close to the amorphization pressure of ice we observe a slow increase in the kinetic energy for both hydrogen 
and oxygen, without any appreciable change in its trend. This contrasts with the trend observed for the atomic mean-square displacements $\Delta_{r}^{2}$ shown above in Figs. 6 and 7 . This is a consequence of the fact that $E_{k}$ is related to the spread of the quantum paths, as measured by the mean-square displacements $Q_{r}^{2}$, and not to the centroid motion, which represents displacements of the overall paths, irrespective of their size and shape. The fast increase in $\Delta_{r}^{2}$ close to the spinodal points is mainly due to an increase in the centroid delocalization $C_{r}^{2}$.

\section{SUMMARY}

We have presented results of PIMD simulations of ice Ih in the isothermal-isobaric ensemble at different pressures and temperatures. This kind of simulations have allowed us to study this water phase in the pressure region where it is metastable, and approach the spinodal points at which it becomes mechanically unstable. At $P<0$ we found a spinodal pressure $P_{s}$ ranging from -1.4 to $-0.7 \mathrm{GPa}$ in the temperature range from 50 to $300 \mathrm{~K}$. At positive pressures, we obtained a spinodal in the range $P_{s}^{\prime} \sim 1.1-1.3 \mathrm{GPa}$, that is well defined at temperatures $T \lesssim 100 \mathrm{~K}$. At higher temperatures, kinetic processes favor amorphization of the solid for pressures clearly lower than the estimated spinodal pressure.

Although the molar volume decreases with increasing pressure, the interatomic distances in the solid change in a peculiar way that remembers their temperature dependence. Thus, the distance between oxygen atoms in neighboring molecules decreases for increasing pressure, but the intramolecular $\mathrm{O}-\mathrm{H}$ distance becomes larger for higher pressure. This is a consequence of the hydrogen bonds connecting contiguous molecules, which become stronger as the volume (or $\mathrm{O}-\mathrm{O}$ distance) is reduced, causing a weakening of the intramolecular $\mathrm{O}-\mathrm{H}$ bonds.

For the bulk modulus of ice Ih, we obtain a maximum at $0.3-0.5 \mathrm{GPa}$, which increases slowly as the temperature is lowered. At higher and lower (negative) pressures,
$B$ is found to decrease and extrapolates to zero at the corresponding spinodal pressure $\left(P_{s}\right.$ or $\left.P_{s}^{\prime}\right)$. Close to the spinodals, we observe an increase in the atomic delocalization for both oxygen and hydrogen atoms, especially at temperatures in the order of the melting temperature of ice Ih. For the kinetic energy, however, we do not observe any anomalous effect, and it is found to increase smoothly as temperature or pressure is raised.

We have assessed the importance of quantum effects by comparing results obtained from PIMD simulations with those yielded by classical simulations. Concerning the stability of ice Ih, we have found that quantum effects reduce the metastability region of this solid phase, for both positive and negative pressures. At low temperatures, this means that both spinodal pressures $P_{s}$ and $P_{s}^{\prime}$ are shifted by about $0.3 \mathrm{GPa}$. Structural variables also change when quantum nuclear motion is considered. Thus, the crystal volume, interatomic distances, and atomic delocalization suffer appreciable modifications in the range of temperature and pressure considered here. Most important is the increase in the mean-square displacement of hydrogen, which contributes to weaken the intermolecular $\mathrm{H}$ bonds, and to strengthen the intramolecular $\mathrm{O}-\mathrm{H}$ bonds.

An extension of this work can consist in studying the high-density amorphous phase obtained from ice Ih under pressure, as well as other amorphous water phases, which may show peculiar properties as a function of pressure and temperature. Path-integral molecular dynamics simulations with interatomic potentials similar to that employed here can be also adequate to study these solid phases.

\section{Acknowledgments}

This work was supported by Ministerio de Ciencia e Innovación (Spain) through Grant FIS2009-12721-C04-04 and by Comunidad Autónoma de Madrid through Program MODELICO-CM/S2009ESP-1691.
1 D. Eisenberg and W. Kauzmann, The Structure and Properties of Water (Oxford University Press, New York, 1969).

2 V. F. Petrenko and R. W. Whitworth, Physics of Ice (Oxford University Press, New York, 1999).

${ }^{3}$ F. Franks, Water: A Matrix of Life (Royal Society of Chemistry, London, 2000), 2nd ed.

${ }^{4}$ G. W. Robinson, S. B. Zhu, S. Singh, and M. W. Evans, Water in Biology, Chemistry and Physics (World Scientific, Singapore, 1996).

${ }^{5}$ F. Sciortino, U. Essmann, H. E. Stanley, M. Hemmati, J. Shao, G. H. Wolf, and C. A. Angell, Phys. Rev. E 52, 6484 (1995).

6 O. Mishima, L. D. Calvert, and E. Whalley, Nature 310, 393 (1984).

7 J. S. Tse, D. D. Klug, C. A. Tulk, I. Swainson, E. C. Svens- son, C. K. Loong, V. Shpakov, V. R. Belosludov, R. V. Belosludov, and Y. Kawazoe, Nature 400, 647 (1999).

8 J. S. Tse, D. D. Klug, M. Guthrie, C. A. Tulk, C. J. Benmore, and J. Urquidi, Phys. Rev. B 71, 214107 (2005).

9 R. J. Nelmes, J. S. Loveday, T. Strässle, C. L. Bull, M. Guthrie, G. Hamel, and S. Klotz, Nature Phys. 2, 414 (2006).

10 J. A. Barker and R. O. Watts, Chem. Phys. Lett. 3, 144 (1969).

11 A. Rahman and F. H. Stillinger, J. Chem. Phys. 55, 3336 (1971).

12 M. W. Mahoney and W. L. Jorgensen, J. Chem. Phys. 115, 10758 (2001).

13 Y. Koyama, H. Tanaka, G. Gao, and X. C. Zeng, J. Chem. Phys. 121, 7926 (2004).

${ }^{14}$ W. L. Jorgensen and J. Tirado-Rives, Proc. Natl. Acad. 
Sci. USA 102, 6665 (2005)

15 J. L. F. Abascal and C. Vega, J. Chem. Phys. 123, 234505 (2005).

16 F. Paesani, W. Zhang, D. A. Case, T. E. Cheatham, and G. A. Voth, J. Chem. Phys. 125, 184507 (2006).

17 C. McBride, C. Vega, E. G. Noya, R. Ramírez, and L. M. Sesé, J. Chem. Phys. 131, 024506 (2009).

18 B. Chen, I. Ivanov, M. L. Klein, and M. Parrinello, Phys. Rev. Lett. 91, 215503 (2003).

19 M. V. Fernández-Serra and E. Artacho, Phys. Rev. Lett. 96, 016404 (2006).

20 J. A. Morrone and R. Car, Phys. Rev. Lett. 101, 017801 (2008).

21 S. Yoo, X. C. Zeng, and S. S. Xantheas, J. Chem. Phys. 130, 221102 (2009).

22 J. Wang, G. Roman-Perez, J. M. Soler, E. Artacho, and M. V. Fernandez-Serra, J. Chem. Phys. 134, 024516 (2011).

23 B. Kolb and T. Thonhauser, Phys. Rev. B 84, 045116 (2011)

${ }^{24}$ O. Akin-Ojo and F. Wang, Chem. Phys. Lett. 513, 59 (2011).

25 M. J. Gillan, Phil. Mag. A 58, 257 (1988).

26 D. M. Ceperley, Rev. Mod. Phys. 67, 279 (1995).

27 E. Sanz, C. Vega, J. L. F. Abascal, and L. G. MacDowell, Phys. Rev. Lett. 92, 255701 (2004).

28 R. Ramírez and C. P. Herrero, J. Chem. Phys. 133, 144511 (2010)

29 H. Gai, G. K. Schenter, and B. C. Garrett, J. Chem. Phys. 104, 680 (1996).

${ }^{30}$ L. Hernández de la Peña, M. S. Gulam Razul, and P. G. Kusalik, J. Chem. Phys. 123, 144506 (2005).

31 L. Hernández de la Peña and P. G. Kusalik, J. Chem. Phys. 125, 054512 (2006).

32 F. Paesani and G. A. Voth, J. Phys. Chem. C 112, 324 (2008).

33 T. E. Markland, J. A. Morrone, B. J. Berne, K. Miyazaki, E. Rabani, and D. R. Reichman, Nature Phys. 7, 134 (2011).

34 S. Habershon, T. E. Markland, and D. E. Manolopoulos, J. Chem. Phys. 131, 024501 (2009).

35 C. P. Herrero and R. Ramírez, J. Chem. Phys. 134, 094510 (2011)

36 R. P. Feynman, Statistical Mechanics (Addison-Wesley, New York, 1972).

37 H. Kleinert, Path Integrals in Quantum Mechanics, Statistics and Polymer Physics (World Scientific, Singapore, 1990).

38 G. J. Martyna, M. E. Tuckerman, D. J. Tobias, and M. L. Klein, Mol. Phys. 87, 1117 (1996).

39 M. E. Tuckerman and A. Hughes, in Classical and Quantum Dynamics in Condensed Phase Simulations, edited by B. J. Berne, G. Ciccotti, and D. F. Coker (Word Scientific, Singapore, 1998), p. 311.

40 M. E. Tuckerman, in Quantum Simulations of Complex Many-Body Systems: From Theory to Algorithms, edited by J. Grotendorst, D. Marx, and A. Muramatsu (NIC, Jülich, 2002), p. 269.
41 V. Buch, P. Sandler, and J. Sadlej, J. Phys. Chem. B 102, 8641 (1998).

42 B. S. Gonzalez, E. G. Noya, C. Vega, and L. M. Sese, J. Phys. Chem. B 114, 2484 (2010).

43 T. F. Miller and D. E. Manolopoulos, J. Chem. Phys. 123, 154504 (2005).

44 D. C. Ginnings and R. J. Corruccini, J. Res. Natl. Bur. Stand. 38, 583 (1947).

45 G. Dantl and I. Gregora, Naturwiss. 55, 176 (1968).

46 T. Strässle, A. M. Saitta, S. Klotz, and M. Braden, Phys. Rev. Lett. 93, 225901 (2004).

47 N. W. Ashcroft and N. D. Mermin, Solid State Physics (Saunders College, Philadelphia, 1976).

48 J. S. O. Evans, J. Chem. Soc., Dalton Trans. p. 3317 (1999).

49 S. M. Bennington, J. Li, M. J. Harris, and D. K. Ross, Physica B 263, 396 (1999).

${ }^{50}$ H. Gai, G. K. Schenter, and B. C. Garrett, Phys. Rev. B 54, 14873 (1996).

51 H. Tanaka and J. Mohanty, J. Am. Chem. Soc. 124, 8085 (2002).

52 R. Feistel and W. Wagner, J. Phys. Chem. Ref. Data 35, 1021 (2006).

53 L. D. Landau and E. M. Lifshitz, Statistical Physics (Pergamon, Oxford, 1980), 3rd ed.

54 C. P. Herrero, J. Phys.: Condens. Matter 20, 295230 (2008).

55 C. P. Herrero and R. Ramírez, Phys. Rev. B 63, 024103 (2000).

56 C. P. Herrero, Phys. Rev. B 68, 172104 (2003).

57 H. J. Maris and Q. Xiong, Phys. Rev. Lett. 63, 1078 (1989).

58 H. J. Maris, Phys. Rev. Lett. 66, 45 (1991).

59 J. Boronat, J. Casulleras, and J. Navarro, Phys. Rev. B 50, 3427 (1994)

60 R. J. Speedy, J. Phys. Chem. 86, 3002 (1982).

61 T. Strässle, S. Klotz, J. S. Loveday, and M. Braden, J. Phys.: Condens. Matter 17, S3029 (2005).

62 J. S. Tse, J. Chem. Phys. 96, 5482 (1992).

63 J. S. Tse and M. L. Klein, Phys. Rev. Lett. 58, 1672 (1987).

64 J. S. Tse and M. L. Klein, J. Chem. Phys. 92, 3992 (1990).

65 K. Nygård, M. Hakala, S. Manninen, A. Andrejczuk, M. Itou, Y. Sakurai, L. G. M. Pettersson, and K. Hämäläinen, Phys. Rev. E 74, 031503 (2006).

66 W. B. Holzapfel, J. Chem. Phys. 56, 712 (1972).

67 D. D. Klug and E. Whalley, J. Chem. Phys. 81, 1220 (1984).

68 J. M. Besson, P. Pruzan, S. Klotz, G. Hamel, B. Silvi, R. J. Nelmes, J. S. Loveday, R. M. Wilson, and S. Hull, Phys. Rev. B 49, 12540 (1994).

69 M. F. Herman, E. J. Bruskin, and B. J. Berne, J. Chem. Phys. 76, 5150 (1982).

70 R. Ramirez and C. P. Herrero, Phys. Rev. B 84, 064130 (2011).

71 G. F. Reiter, J. C. Li, J. Mayers, T. Abdul-Redah, and P. Platzman, Braz. J. Phys. 34, 142 (2004). 